\title{
PARTICLE SEPARATION OF A MODIFIED FEED NOZZLE HYDROCYCLONE UNDER DIFFERENT OPERATING PARAMETERS
}

\author{
Zsolt Hegyes \\ BSc student, University of Miskolc, Institute of Energy Engineering and Chemical Machinery \\ 3515 Miskolc, Miskolc-Egyetemváros, e-mail: hegyes.zs97@gmail.com \\ Máté Petrik \\ assistant lecturer, University of Miskolc, Institute of Energy Engineering and Chemical Machinery \\ 3515 Miskolc, Miskolc-Egyetemváros, e-mail:vegypet@uni-miskolc.hu \\ Gábor L. Szepesi \\ associate professor, University of Miskolc, Institute of Energy Engineering and Chemical Machinery \\ 3515 Miskolc, Miskolc-Egyetemváros, e-mail: szepesi@uni-miskolc.hu
}

\begin{abstract}
During the operation of the hydrocyclone the cut size diameter is the most important data. This is connected to feed rate, which is closely related to the feed cross section. Preliminary research has revealed that square cross-section is more effective than circular cross-section. The research compared 2 types of feed cross sections at 5 different feed rates. One is a standard rectangular cross-section and the other is a square cross-section that narrows with a baffle plate. Preliminary calculations for cut size diameter have shown that better particle separation at all speeds can be achieved with the baffle plate solution. In both types, the increased velocity created decreased cut size diameter. During the simulation, the baffle plate did not cause any abnormalities in the internal pressure and velocity distributions. The simulation revealed that the particles did not behave as previously calculated.
\end{abstract}

Keywords: hydrocyclone, particle separation, feed inlet

\section{Introduction}

In various industrial fields such as mining, agriculture, water supply is required for various purposes. These waters are usually pumped out from natural sources, from rivers or lakes. However, with this type of water supply, there is a risk that other undesirable substances (for example sand or oil) will enter the system. These solid contaminants when entering tubes or valves, cause abrasive damage, clogging, and possibly improper operation of a particular operation in the machines and the pipelines that connect them.

There are several devices are available to avoid the dangers describe above. Examples are various settlers, filters, centrifuges and so on. These devices are excellent to separate inhomogeneous mixtures. The purpose of the separation may be, in addition to water purification, to recover solid particles, possibly to extract oil from water or other liquid media. However, each of these devices has number of disadvantages that make them difficult to operate. Centrifuges are expensive to operate (maintenance, power consumption, etc.), filters are intermittent (many overtime), and settlers are very space consuming.

Nowadays, environmental friendly and cost-effective operation are the most important aspect in industrial systems. The hydrocyclone can be a solution for these operating problems. The hydrocyclone 
is suitable for separating any two-component inhomogeneous mixture with extremely high efficiency. A hydrocyclone is a very simple device in itself that is capable of separating large amounts of mixtures relative to its size. These devices are useful in pre-mechanical filtration operations as they significantly reduce the load on the filters and are also suitable for thickening even dilute suspensions.

\section{Hydrocyclone structue and principle of operation}

The hydrocyclone consist of 5 different elements: feed nozzle (1), upper cylinder part (2), cone (3), spigot (4), vortex finder (5). The structure of a general cyclone is shown in Figure 1.

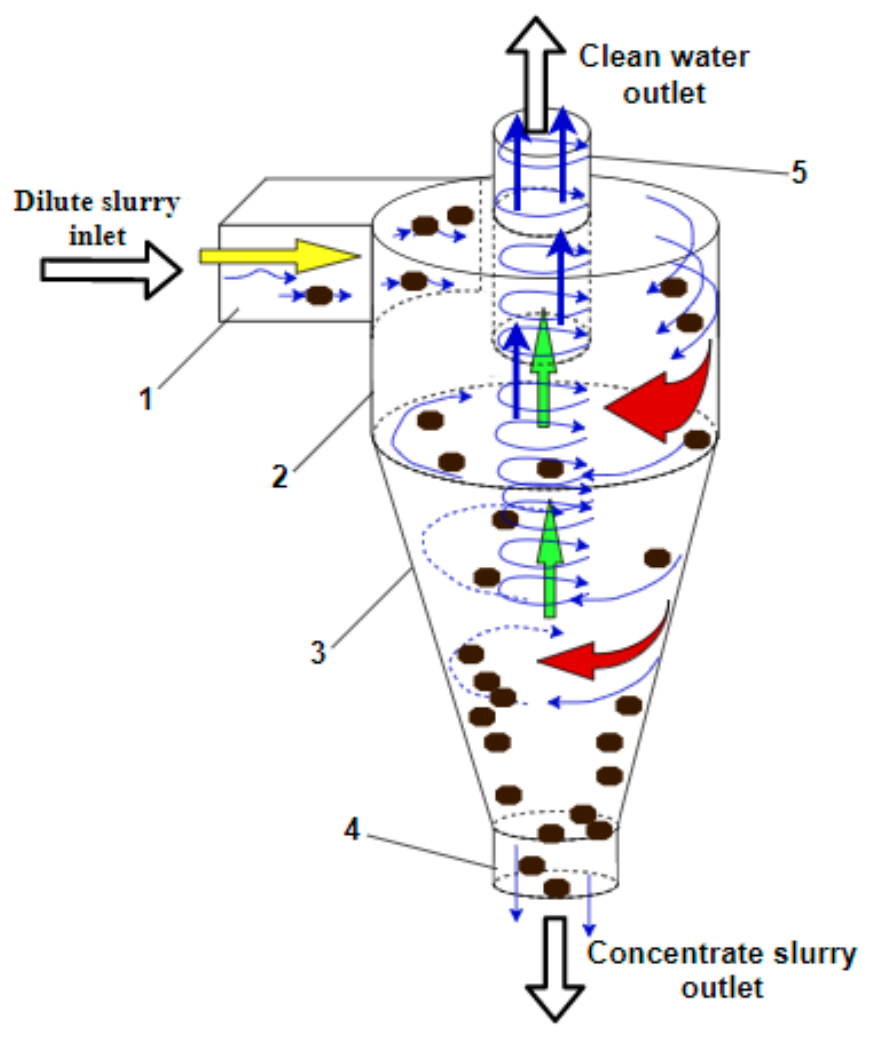

Figure 1. Hydrocylone structure and principle operation

Researchers have already performed a number of geometric optimization methods on cyclones to increase the separation efficiency. To understand the separation process, the flow processes in the cyclone need to understand (Figure 1).

The mixture to be separated enters in the feed nozzle for which the required pressure is provided by a pump. The mass flow of the feed is a constant value. The hydrocyclone may only be installed on the pressed side after the pump. The feed enters in a straight line (tangentially to the cylindrical shell; yellow arrow). As the liquid leaves the nozzle, the tangential motion changes to a circular motion (red arrow), as gravity also affects the fluid and the particles in it. The motion of the flowing liquid can thus be divided into 3 vector components: tangential- $\left(v_{\theta}\right)$, axial- $\left(v_{a}\right)$, and radial velocity component $\left(v_{r}\right)$, where the tangential component is the determining factor for the operation of the hydrocyclone (Figure 2 ). 


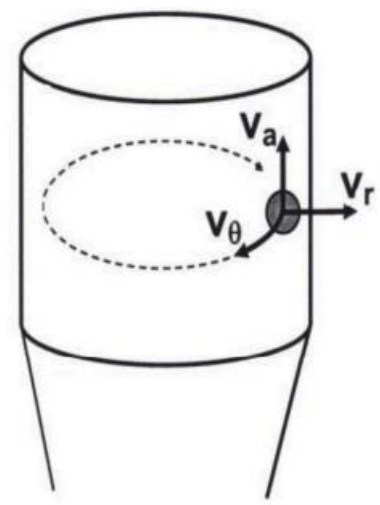

Figure 2. Velocity components in the hydrocyclone

The moving fluid travels further and lower in a spiral path towards the outlet nozzle through the conical part while being subjected to a centrifugal force. As a result of the centrifugal force, the heavier particles adhere to the wall of the cyclone and accumulate there (in the investigated case it is sand), while the lighter component begins to accumulate in the middle of the cyclone (water). The difference in density of the components in the cyclone is the driving force behind the separation. This type of mechanism is described by Stokes' law (Eq 1), which applies to a centrifugal force field (Van Duijn, Riemeta, 1983).

$$
v(r)=\frac{d_{50}^{2} \cdot\left(\rho_{s}-\rho_{l}\right)}{18 \cdot \eta_{l}} \cdot \frac{v_{\theta}^{2}}{r}
$$

As the diameter of the conical portion decreases, as it travels downward, while the mass flow is constant, the circumferential velocity of the liquid increases, resulting in greater centrifugal force and intensified separation. The pressure inside the cyclone starts to increase, which is the largest along the wall, and decreases towards the inside of the cyclone until it reaches the outside atmospheric pressure. The pressure difference measured between the lower outlet nozzle and the vortex search nozzle, which is the driving force of the flow. This forces the lighter phase to flow towards the vortex search tube (green arrow). This flow occurs as a ,tubular" vortex in the centerline of the cyclone, the motion of which also occurs in a helical and axial trajectory. The direction of the spiral motion is the same as the direction of the external spiral motion. However, the direction of the axial movement is opposite to the direction of flow of the bottom product exits through the outlet nozzle. The liquid escapes through the vortex nozzle can be drained into a tank or pool. It may be that the effluent still contains small particles, the concentration of these particles depending on the efficiency of the cyclone (Eq 3). The flow of the slurry in the cyclone (in the feed nozzle) should always be turbulent, which can be characterized by the Reynolds number (Eq 2) (Aldrich, 2015).

$$
\operatorname{Re}=\frac{v \cdot D \cdot \rho_{l}}{\eta_{l}}
$$

\section{Separation efficiency of the hydrocyclone}

The efficiency of a hydrocyclone is affected by several factors. These may consist of some geometric modification or any change in the feed medium (speed/pressure, viscosity etc.). Hydrocyclone 
optimization has been addressed by several researchers who have achieved significant results with their research that proved essential for later design. The efficiency of the hydrocyclone can be interpreted according to the following formula (Dwari et al., 2004):

$$
\eta \%=\frac{W \%_{\text {inlet }}-W \%_{\text {outlet }}}{W \%_{\text {inlet }}} \cdot 100
$$

Geometry:

The development of a well-coordinated geometry is essential for the proper operation of the hydrocyclone. The geometric optimization of the hydrocyclone has been addressed by several researchers (Statie et al., 2001; Chu et al., 2000; Delgadillo, Rajamani, 2007). In general, it has been found that the most important geometry in cyclone operation is the vortex search tube and the diameter and length of the cross-section of the nozzle (Rocha et al., 2020) (feed angle). The effect of its geometric dimensions is shown in Table 1.

Table 1. Geometric proportions of hydrocyclones (Aldrich, 2015)

\begin{tabular}{|l|c|c|c|}
\hline Factor & Throughput $(\mathbf{Q})$ & Cut size $\left(d_{50}\right)$ & $\begin{array}{c}\text { Sharpness of } \\
\text { classification }\end{array}$ \\
\hline Cyclone diameter & + & + & + \\
\hline Feed inlet & + & - & - \\
\hline Vortex finder diameter & + & + & + \\
\hline Spigot diameter & + & - & - \\
\hline Cone angle & N/A & + & + \\
\hline Free vortex finder height & + & + & + \\
\hline
\end{tabular}

The effects of cyclone geometry (summarized in Table 1) and operational variables on the throughput, cut size, and sharpness of classification in hydrocyclones with „," indicating an increase and ,-" indicating a decrease in the performance criterion with an increase in the factor (Table 1) (Aldrich, 2015).

Particle size and pressure drop:

The hydrocyclone pressure drop is interpreted as difference between the inlet and the outlet pressure of the cyclone (Eq 4) (Lv et al., 2020).

$$
\Delta P=P_{i}-P_{o}
$$

By increasing the particle size, the efficiency improves, by increasing the higher feed pressure, this increase is exponential. In all particle size ranges, an increase in pressure drops leads to an improvement in efficiency.

Particle shape factor:

The shape factor greatly influences the separation efficiency. As sphericity (deviation from sphere) decreases, so does the separation efficiency (Abdollahzadeh, 2015).

Velocity Inlet and concentration:

Increasing the rate is favourable for the separation efficiency, especially at higher concentrations. it is important to choose the appropriate feed concentration (Li et al., 2020).

Viscosity:

Viscosity greatly affects the separation of the particles in the flowing liquid. As the viscosity increases, this ability decreases. As the viscosity increases, which degrades efficiency (Marthinussen, 2014). 


\section{Description of investigation}

During this research, I performed simulation studies on a hydrocyclone created using the geometric ratios used by Rietema (Tang et al., 2017). The simulation conditions were the same as the test conditions used by Yan Xia (Tang et al., 2017), but at this case the density was $1600 \mathrm{~kg} / \mathrm{m}^{3}$. It is a square inlet type cyclone with diameter of $75 \mathrm{~mm}$. Since research prove that the feed with a rectangular cross section proves to be better than the square cross section (Rocha et al., 2020), a rectangular cross section was investigated in a novel way. The modification was achieved by installing a baffle with a radius $R=172 \mathrm{~mm}$. The aim of the research is to investigate whether this type of cross-sectional constriction causes flow disturbances in the cyclone. This cyclone corresponds to the Cyclone B described in Figure 3. Not only the cyclone with the baffle plate, but also a design that correspond to the extension of the narrowed cross-section were investigated. This cyclone corresponds to the Cyclone A. The formation of the cyclone is shown in Figure 3. The sizes of the geometries are summarized in Table 2.

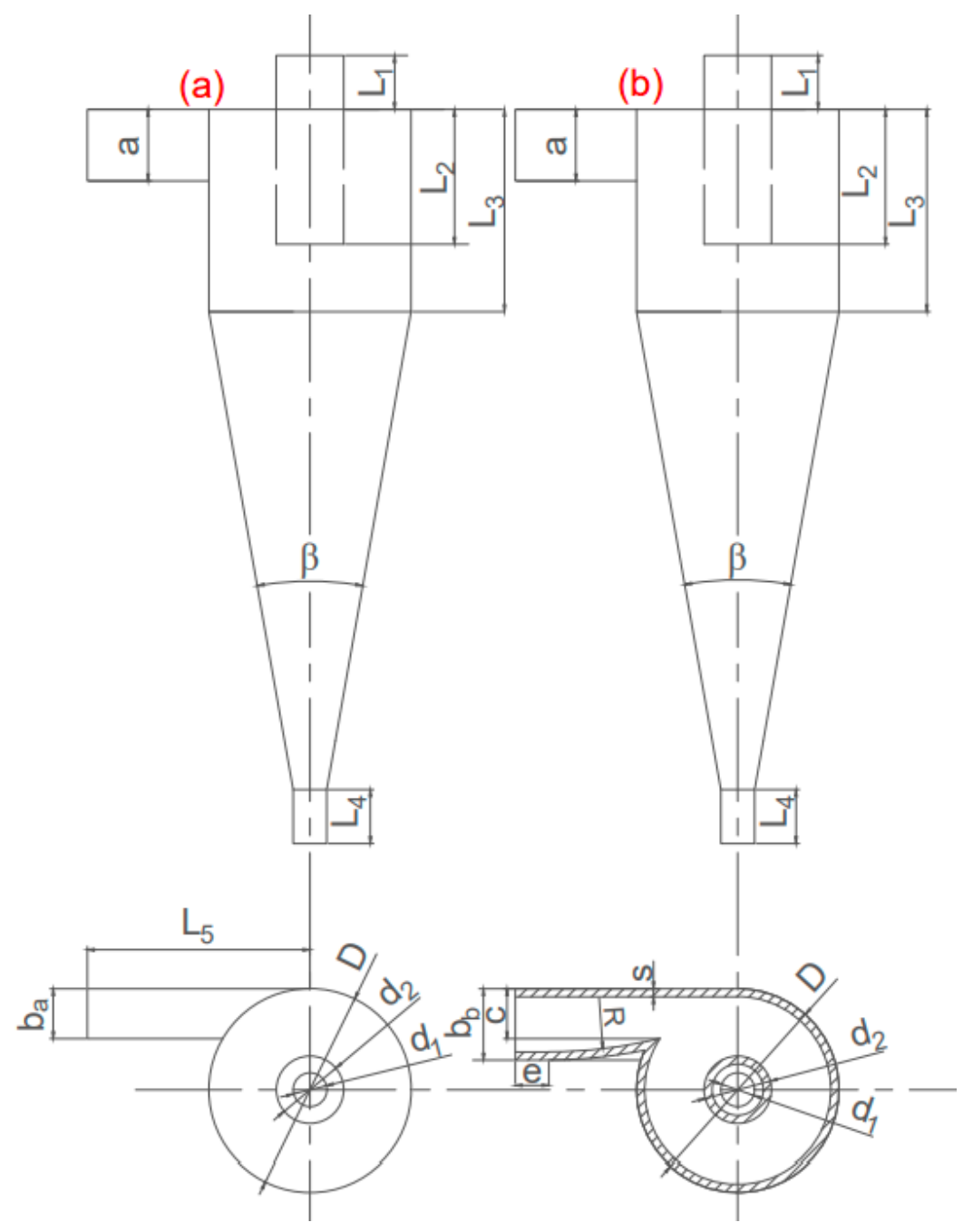

Figure 3. The dimension of the hydrocyclones 
Table 2. The dimensions of the cyclones

\begin{tabular}{|l|l|l|l|l|l|l|l|l|l|l|l|l|l|l|l|}
\hline & $\boldsymbol{L}_{\boldsymbol{1}}$ & $\boldsymbol{L}_{\boldsymbol{2}}$ & $\boldsymbol{L}_{\boldsymbol{3}}$ & $\boldsymbol{L}_{\boldsymbol{4}}$ & $\boldsymbol{D}$ & $\boldsymbol{b}_{\boldsymbol{a}}$ & $\boldsymbol{b}_{\boldsymbol{b}}$ & $\boldsymbol{b}_{\boldsymbol{c}}$ & $\boldsymbol{c}$ & $\boldsymbol{d}_{\boldsymbol{1}}$ & $\boldsymbol{d}_{2}$ & $\boldsymbol{e}$ & $\boldsymbol{s}$ & $\boldsymbol{R}$ & $\boldsymbol{\beta}$ \\
\hline $\begin{array}{l}\text { a)- } \\
\text { cyclone }\end{array}$ & 20 & 50 & 75 & 20 & 75 & 18,5 & - & - & - & 12,5 & 25 & - & 3 & - & 20 \\
\hline $\begin{array}{l}\text { b)- } \\
\text { cyclone }\end{array}$ & 20 & 50 & 75 & 20 & 75 & - & 26,5 & - & 18,5 & 12,5 & 25 & 12,5 & 3 & 172 & 20 \\
\hline
\end{tabular}

\subsection{Simulation parameters}

The cyclone used by Rietema (Tang et al., 2017) did not have data on the magnitude of the wall thickness, so it is assumed to $3 \mathrm{~mm}$. This data plays an important role in the formation of the internal flow space. The simulations performed with a program called SC/Tetra, where the following conditions and parameters were set:

- Type of the model net:

- Number of elements

- Flow type:

- Applied turbulent model:

- Times step:

- Number of cycles:

- Time of one experiment:

- Temperature in the cyclone:

- Solid density (sand):

- Water density:

- Viscosity of the water:

- Thermal conductivity of the water

- Foluid type:

- Used inlet velocities

- Used particle sizes: $60 \mu \mathrm{m}, 80 \mu \mathrm{m}, 100 \mu \mathrm{m}$ tetragonal

420453-559465

turbulent

$\mathrm{k}-\Omega$ model

$0,5 \mathrm{~s}$

3000

$30 \mathrm{~s}$

$20{ }^{\circ} \mathrm{C}$

$1600 \mathrm{~kg} / \mathrm{m}^{3}$

$998,2 \mathrm{~kg} / \mathrm{m}^{3}$

0,001016 Pas

$0,5991 \mathrm{~W} / \mathrm{mK}$

incompressible

$1,28 \mathrm{~m} / \mathrm{s} ; 1,78 \mathrm{~m} / \mathrm{s} ; 2,28 \mathrm{~m} / \mathrm{s} ; 2,78 \mathrm{~m} / \mathrm{s} ; 3,03 \mathrm{~m} / \mathrm{s}$

$5 \mu \mathrm{m}, 10 \mu \mathrm{m}, 15 \mu \mathrm{m}, 20 \mu \mathrm{m}, 25 \mu \mathrm{m}, 30 \mu \mathrm{m}, 40 \mu \mathrm{m}$,

\subsection{Velocity distributions}

Two significant velocity components in the cyclone flow were investigated. The y-direction tangential velocity and the z-direction axial velocity. During particle separation, these two velocity components are dominant (Aldrich, 2015). It can be clearly seen from the tangential velocity figure (Figure 4) that the highest tangential velocity (in absolute value) occurs along the outer wall of the cyclone, while the smallest occurs in the middle of the cyclone. Considering the middle swirling current, the current formed in b)-cyclone can be considered more stable. Significant fluctuations are observed in the lower part of cyclone a), which can have a detrimental effect on cyclone operation as it affects stable flow and separation (Li et al., 2020). 


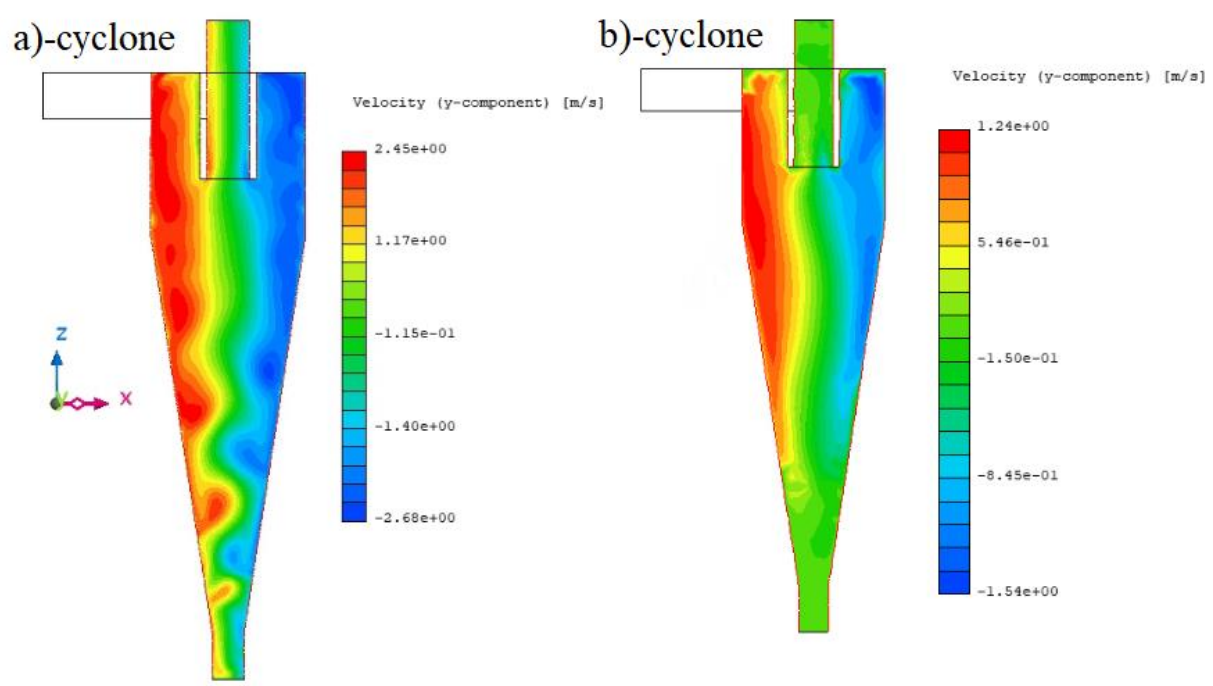

Figure 4. Velocity distribution in the y-direction

At axial velocities, an increase in velocity toward the centerline of the cyclone is observed, reaching its maximum value in the vortex finder (see Figure 5). I found a significant difference in the velocity profiles of cyclone a) and b). The a)-cyclone shows the upward flow of material in the middle, while cyclone b) does not. For cyclone b), it can be observed that the upward axial velocity occurs only at the vortex finder, which affects particle deposition (see Figure 5). The line where the axial velocity changes direction is called LZVV (Line of Locus Zero Vertical Velocity). The speed in this line is $0 \mathrm{~m} / \mathrm{s}$ (Bradley, 1965).

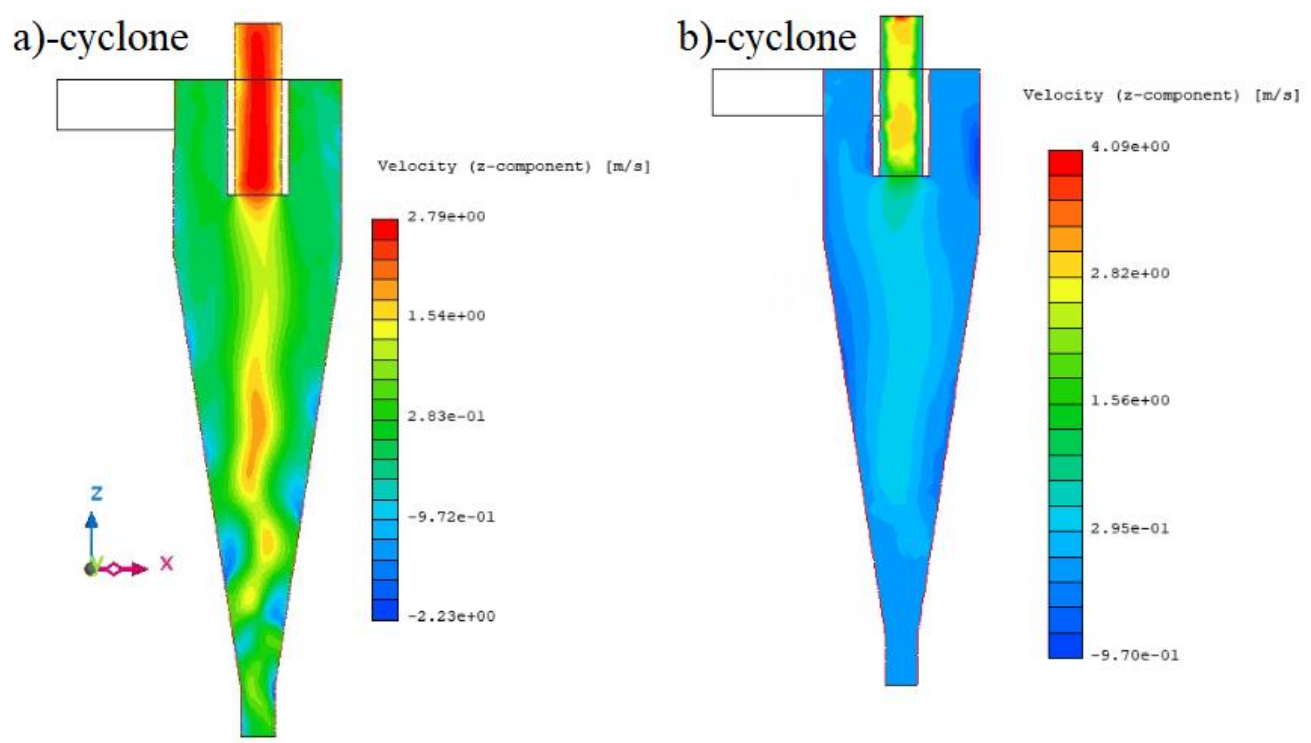

Figure 5. Velocity distribution in the z-component 


\subsection{Pressure distributions}

It can be clearly seen from Figure 6 that the different inlet designs influence the internal pressure value. For all two cyclones, an increasing pressure value is observed moving from the centerline of the cyclone to the outside of the cyclone. This upward trend is greatest for a)-cyclone In the case of a)-cyclone, a negative pressure value is also observed in the centerline of the cyclone. This is possible because the upward vortex formed in the centerline of the cyclone exerts a suction effect that appears as a negative pressure relative to the pressure in the cyclone (Bradley, 1965).

The pressure difference between the inlet and the vortex finder can be clearly seen in Figure 6 . Comparing a)-cyclone to b)-cyclone, it can be seen that a larger pressure difference can be achieved with the feed nozzle used for a)-cyclone than with the nozzle used for a)-cyclone This is related to higher tangential velocities (Rocha et al., 2020).

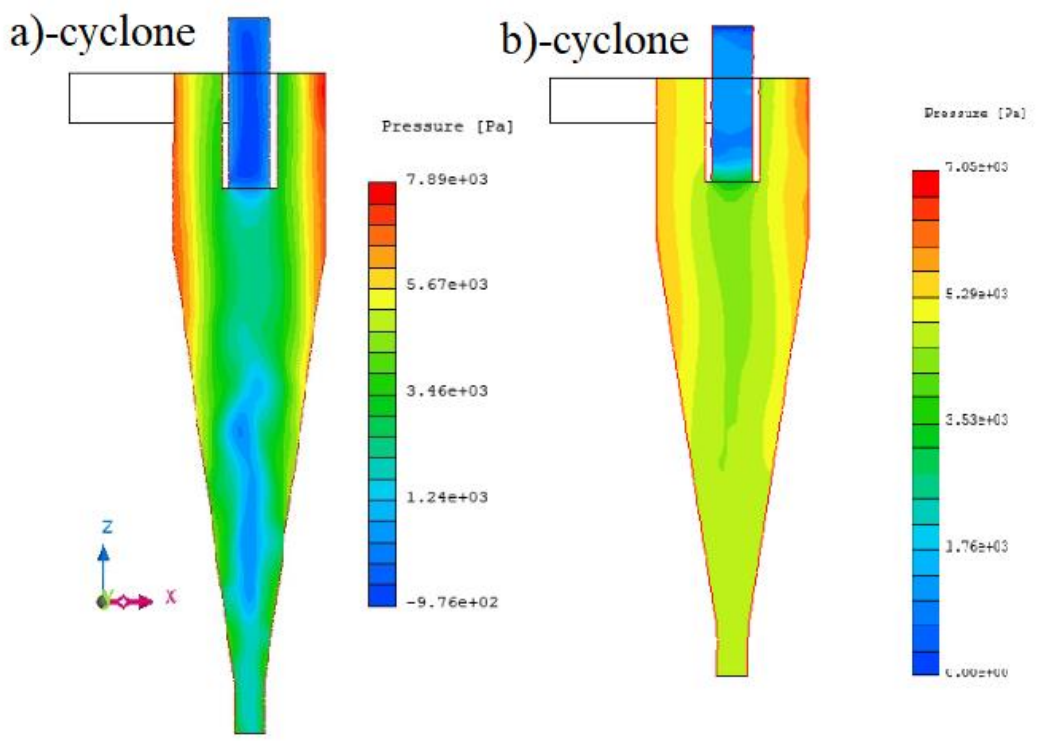

Figure 6. Pressure distribution in the $x$-z plane

\subsection{Particle separation}

One of the most important features of hydrocyclones is the cut size diameter, which can still be $100 \%$ separated. This resulted in a number of formulas, which D. Bradley (Bradley, 1965) collected in detail. I performed preliminary calculations for the cut size diameters in order to compare them with later simulation results. For this, the formula created by Yoshiuka and Hotta (Eq 5) is used, as all the data in the formula are available to me or can be calculated.

$$
d_{50}=6,3 \cdot 10^{6} \cdot\left(\frac{\eta_{l}}{\rho_{s}-\rho_{l}}\right)^{0,5} \cdot \frac{D^{0,1} \cdot D_{i}^{0,6} \cdot d_{2}^{0,8}}{Q^{0,5}}
$$

Since in the simulation not the volume flow but the velocity was given as an input parameter, the volume flow $(Q)$ must be calculated, which is obtained from the continuity equation (Eq 6). 


$$
Q=v \cdot A=v \cdot b \cdot a
$$

The calculated volume flow values are collected in Table 3 for each five inlet velocities:

Table 3. Volume flows of the hydrocyclones

\begin{tabular}{|l|c|c|c|c|c|}
\hline inlet velocities $[\mathrm{m} / \mathrm{s}]$ & $\mathbf{1 , 2 8}$ & $\mathbf{1 , 7 8}$ & $\mathbf{2 , 2 8}$ & $\mathbf{2 , 7 8}$ & $\mathbf{3 , 0 3}$ \\
\hline$Q$ a)-cyclon $\left[\mathrm{m}^{3} / \mathrm{s}\right]$ & 0,000328 & 0,000456 & 0,000584 & 0,000712 & 0,000776 \\
\hline$Q$ b)-cyclon $\left[\mathrm{m}^{3} / \mathrm{s}\right]$ & 0,000538 & 0,000748 & 0,00958 & 0,001168 & 0,001273 \\
\hline$Q_{l}$ a)-cyclon $[1 / \mathrm{s}]$ & 0,328 & 0,456 & 0,585 & 0,712 & 0,776 \\
\hline$Q_{l}$ b)-cyclon $[1 / \mathrm{s}]$ & 0,538 & 0,748 & 0,958 & 1,168 & 1,273 \\
\hline
\end{tabular}

The $D_{i}$ in Eq 4 refers to the inlet diameter at circle-section inlet type. However, the inlet type was a cross-section style, so equivalent dimeter must be calculated according to Eq. 7.

$$
D_{i}=\sqrt{\frac{4 \cdot a \cdot b}{\pi}}
$$

Based in the Equation 4, the following cut size diameters are get:

Table 4. Cut size diameters

\begin{tabular}{|l|c|c|c|c|c|c|}
\hline inlet velocities $[\mathrm{m} / \mathrm{s}]$ & $\mathbf{1 , 2 8}$ & $\mathbf{1 , 7 8}$ & $\mathbf{2 , 2 8}$ & $\mathbf{2 , 7 8}$ & & $\mathbf{3 , 0 3}$ \\
\hline$d_{50}$ a)-cyclon $[\mu \mathrm{m}]$ & 41,31 & 35,03 & 30,95 & 28,03 & & 26,85 \\
\hline$d_{50}$ b)-cyclon $[\mu \mathrm{m}]$ & 32,26 & 27,36 & 24,17 & 21,89 & & 20,97 \\
\hline
\end{tabular}

It can be clearly seen from Table 4 that the increasing velocity value occurs a decreasing cut size diameter. This phenomenon can be well seen in both cyclones. For b)-cyclone this value is one to 6 to $10 \mu \mathrm{m}$ less than the cut-off values for a)-cyclone each velocity range. This was due to the speedincreasing effect of the narrowed cross-section. However, this type of calculation cannot be considered fully authoritative, as the supply pressure and possible flow disturbances have been disregarded here. The simulation results related to particle separation are shown in Figure 7.

The results of the simulation show that the particles in the cyclones did not behave as usual. The particles collected in the middle or lower third of the cyclone and circulated in a circular orbit. The increased number of cycles and refined settings did not change the result either.

Possible causes of the particle behaviour:

- In the cyclone, the amplification of the return flows at the vortex finder (Zhao et al., 2019) results in too high an upward velocity (Figure 5 b)-cyclone) thus the upward and downward velocities balance each other out.

- The density difference between water and sand is too small.

- Since the cyclone described by Yan-xia et al. (Tang et al., 2017) did not have a wall thickness value, the assumed wall thickness of $3 \mathrm{~mm}$ already means a narrowing on the spigot that is presumably no longer able to remove the leaving slurry at a sufficient rate. 

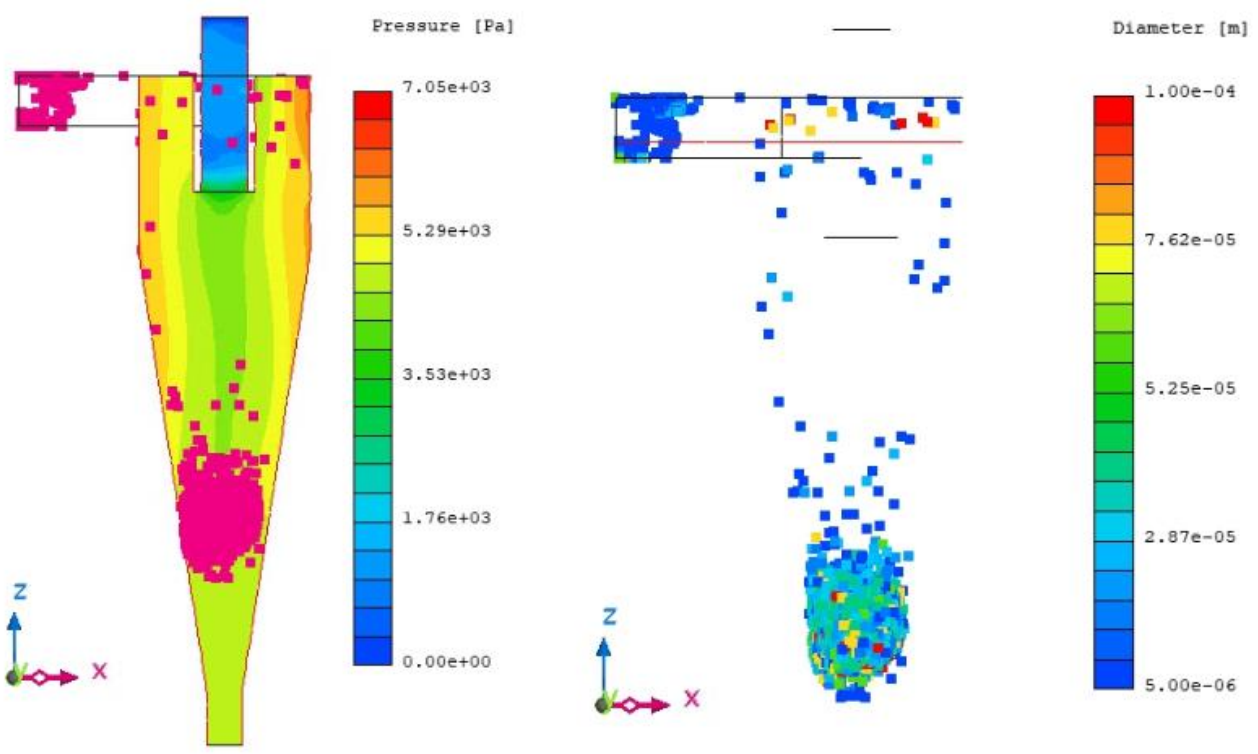

Figure 7. Particle boundary in the b)-cyclone

\section{Conclusion}

In this article I compared 2 hydrocyclones with feed nozzles of different geometries. It can be deduced from the simulation that the pressure and velocity conditions of a)- and b)-cyclone follow the values already represented by the researchers. Despite the established pressure and velocity conditions, I have experienced extremely high fluctuations, which had an influence on the deposition of the particles. This fluctuation caused an increase in residence time to an extent that prevented the particles from leaving the cyclone.

Particle separation occurred because no or very little particle exited the hydrocyclone through the vortex finder, however, the residence time of the particles was so large that it prevented the particles from leaving the lower outlet.

\section{Acknowledgement}

The research was supported by the Hungarian National Research, Development and Innovation Office under the project number K 134358.

\section{Nomenclature}

\section{Latin letters}

A feed inlet area

$\left[\mathrm{m}^{2}\right]$

heighth of the inlet

$[\mathrm{mm}]$

$a \quad$ width of the inlet (ind

$[\mathrm{mm}]$

ex $a$ and $b$ means the cyclone

[mm]

$c$ buffle distance from the inlet region

$D \quad$ diameter of the upper cylinder part

[mm]

$D_{i} \quad$ inlet diameter

$d_{l} \quad$ diameter of the spigot

[m]

[mm] 
$d_{2} \quad$ diameter of the vortex finder

$d_{50} \quad$ cut size diameter

$e \quad$ intrusion of the buffle

$g$ gravitational acceleration

$L_{1} \quad$ length of the vortex finder outside of the cyclone

$L_{2} \quad$ length of the vortex finder inside of the cyclone

$L_{3} \quad$ length of the upper cylinder part

$L_{5} \quad$ legth of the inlet

$P_{i} \quad$ Inlet pressure

$P_{o} \quad$ Overlflow pressure

$\triangle P \quad$ Pressure drop

$Q \quad$ volume flow

$Q_{l} \quad$ volume flow

Re Reyndolds number

$r \quad$ radius of the upper cilynder part

$s \quad$ wall thickness of the cyclone

$v \quad$ inlet velocity

$v_{a} \quad$ axial velocity

$v_{r} \quad$ radial velocity

$v_{\theta} \quad$ tangential velocity

$W \%_{i} \quad$ mass rate for the, i, component

\section{Greek letters}

$\beta \quad$ cone angle

$\eta_{l} \quad$ dynamical viscosity of the liquid phase

$\eta \% \quad$ separation effency

$\rho_{s} \quad$ solid phase density

$\rho_{l} \quad$ liquid phase density [mm]

[m]

[mm]

$\left[\mathrm{m} / \mathrm{s}^{2}\right]$

[mm]

[mm]

[mm]

[mm]

[Pa]

[Pa]

[Pa]

$\left[\mathrm{m}^{3} / \mathrm{s}\right]$

[1/s]

[-]

[m]

[mm]

$[\mathrm{m} / \mathrm{s}]$

[m/s]

$[\mathrm{m} / \mathrm{s}]$

$[\mathrm{m} / \mathrm{s}]$

$\left[\mathrm{kg} / \mathrm{m}^{3}\right]$

$\left.{ }^{\circ}\right]$

[Pas]

[\%]

$\left[\mathrm{kg} / \mathrm{m}^{3}\right]$

$\left[\mathrm{kg} / \mathrm{m}^{3}\right]$

\section{References}

[1] Van Duijn, G., Rietema, K. (1983). Performance of a large-cone-angle hydrocyclone-I: Hydrodynamics. Chemical Engineering Science, 38(10), 1651-1661. https://doi.org/10.1016/0009-2509(83)85022-2

[2] Aldrich, C. (2015). Chapter One - Hydrocyclones. In S. Tarleton (Ed.), Progress in Filtration and Separation (pp. 1-24). Academic Press. https://doi.org/10.1016/B978-0-12-3847461.00001-X

[3] Dwari, R. K., Biswas, M. N., Meikap, B. C. (2004). Performance characteristics for particles of sand FCC and fly ash in a novel hydrocyclone. Chemical Engineering Science, 59(3), 671-684. https://doi.org/10.1016/j.ces.2003.11.015

[4] Statie, E. C., Salcudean, M E., Gartshore, I. S. (2001). The influence of hydrocyclone geometry on separation and fibre classification. Filtration \& Separation, 38(6), 36-41. https://doi.org/10.1016/S0015-1882(01)80380-3

[5] Chu, L.-Y., Chen, W.-M., Lee, X-Z. (2000). Effect of structural modification on hydrocyclone performance. Separation and Purification Technology, 21(1-2), 71-86. https://doi.org/10.1016/S1383-5866(00)00192-1 
[6] Delgadillo, J. A., Rajamani, R. K. (2007). Exploration of hydrocyclone designs using computational fluid dynamics. International Journal of Mineral Processing, 84(1-4), 252-261. https://doi.org/10.1016/j.minpro.2006.07.014

[7] Rocha, C. A. O., Ullmann, G., Silva, D. O., Vieir, L. G. M. (2020). Effect of changes in the feed duct on hydrocyclone performance. Powder Technology, 374, 283-289. https://doi.org/10.1016/j.powtec.2020.07.001

[8] Lv, W.-j., Dang, Z.-h., He, Y., Yu-long Chang, Y.-l. (2020). UU-type parallel mini-hydrocyclone group for oil-water separation in methanol-to-olefin industrial wastewater. Chemical Engineering and Processing - Process Intensification, 149, 107846. https://doi.org/10.1016/j.cep.2020.107846

[9] Abdollahzadeh, L., Habibian, M., Rouhollah, E. (2015). Study of particle's shape factor, inlet velocity and feed concentration on mini-hydrocyclone classification and fishhook effect. Powder Technology, 283, 294-301. https://doi.org/10.1016/j.powtec.2015.05.007

[10] Li, F., Liu, P., Yang, X., Zhang, Y. Y. (2020). Effects of inlet concentration on the hydrocyclone separation performance with different inlet velocity. Powder Technology, 375, 337-351. https://doi.org/10.1016/j.powtec.2020.07.110

[11] Marthinussen, S.-A., Chang, Y.-F., Balak, B. (2014). Removal of particles from highly viscous liquids with hydrocyclones. Chemical Engineering Science, 108, 169-175. https://doi.org/10.1016/j.ces.2014.01.008

[12] Tang, B., Xu, Y.-x., Song, X.-f., Sun, Z. J.-g. (2017). Effect of inlet configuration on hydrocyclone performance. Transactions of Nonferrous Metals Society of China, 27(7), 16451655. https://doi.org/10.1016/S1003-6326(17)60187-0

[13] Bradley, D. (1965). The Hydrocyclone. New York, Pergamon Press Ltd.

[14] Zhao, Q., Cui, B., Wei, D., Song, T. Y. (2019). Numerical analysis of the flow field and separation performance in hydrocyclones with different vortex finder wall thickness. Powder Technology, 345, 478-491. https://doi.org/10.1016/j.powtec.2019.01.030 